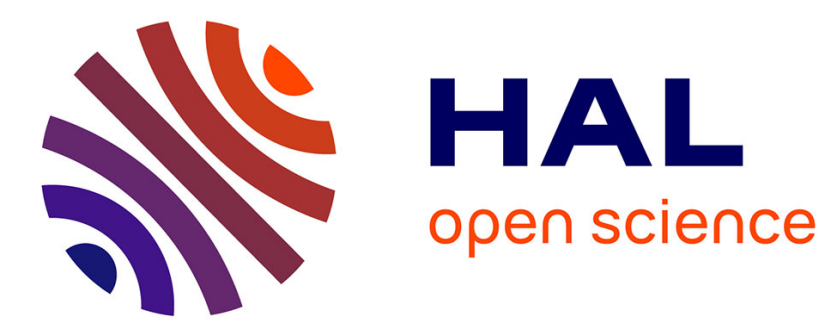

\title{
Plasma-wall self-organization in magnetic fusion
}

Dominique Escande, F Sattin, Paolo Zanca

\section{To cite this version:}

Dominique Escande, F Sattin, Paolo Zanca. Plasma-wall self-organization in magnetic fusion. 2021. hal-03266627v2

\section{HAL Id: hal-03266627 \\ https://hal.science/hal-03266627v2}

Preprint submitted on 30 Jun 2021

HAL is a multi-disciplinary open access archive for the deposit and dissemination of scientific research documents, whether they are published or not. The documents may come from teaching and research institutions in France or abroad, or from public or private research centers.
L'archive ouverte pluridisciplinaire HAL, est destinée au dépôt et à la diffusion de documents scientifiques de niveau recherche, publiés ou non, émanant des établissements d'enseignement et de recherche français ou étrangers, des laboratoires publics ou privés. 


\title{
Plasma-wall self-organization in magnetic fusion
}

\author{
D.F. Escande ${ }^{1}$, F. Sattin ${ }^{2}$ and P. Zanca ${ }^{2}$ \\ ${ }^{1}$ Aix-Marseille Université, CNRS, PIIM, UMR 7345, Marseille, France \\ ${ }^{2}$ Consorzio RFX (CNR, ENEA, INFN, Università di Padova, Acciaierie Venete Spa), \\ Padova, Italy \\ E-mail: dominique.escande@univ-amu.fr
}




\begin{abstract}
.
This paper introduces the concept of plasma-wall self-organization (PWSO) in magnetic fusion. The basic idea is the existence of a time delay in the feedback loop relating radiation and impurity production on divertor plates. Both a zero and a onedimensional description of PWSO are provided. They lead to an iterative equation whose equilibrium fixed point is unstable above some threshold. This threshold corresponds to a radiative density limit, which can be reached for a ratio of total radiated power to total input power as low as $1 / 2$. When detachment develops and physical sputtering dominates, this limit is progressively pushed to very high values if the radiation of non-plate impurities stays low. Therefore, PWSO comes with two basins for this organization: the usual one with a density limit, and a new one with density freedom, in particular for machines using high-Z materials. Two basins of attraction of PWSO are shown to exist for the tokamak during start-up, with a high density one leading to this freedom. This basin might be reached by a proper tailoring of ECRH assisted ohmic start-up in present middle-size tokamaks, mimicking present stellarator start-up. In view of the impressive tokamak DEMO wall load challenge, it is worth considering and checking this possibility, which comes with that of more margins for ITER and of smaller reactors.
\end{abstract}

Keywords plasma-wall interaction, self-organization, sputtering, density limit, radiation Submitted to: Nucl. Fusion

\title{
1. Introduction
}

The existence of a density limit in magnetic fusion plasmas is a concern, since it limits the operational domain of experiments and contributes to the definition of a minimum size of fusion reactors because of Lawson criterion. For the tokamak and the reversed field pinch a simple estimate of this limit, the Greenwald density limit [1], was considered reasonable during two decades. However, this is now strongly challenged. The abstract of a 2013 JET paper [2] states: "In contrary to the well known 'heating power independent Greenwald limit', the L-mode densities limit increases moderately with rising heating power $\left(\sim P^{0.4}\right)$ independently on the wall material". The same scaling with the total heating power $P$ was already present in five papers published between 1986 and 1999 [3, 4, 5, 6, 7]. Furthermore, figure 10 of the recent [8] shows with a database of 5 tokamaks that a scaling like $\left(I P / a^{4}\right)^{4 / 9}$, with $a$ the small radius and $I$ the total current, organizes much better the data than Greenwald's one in $I / a^{2}$; especially device per device.

The new $\left(I P / a^{4}\right)^{4 / 9}$ scaling is a part of those derived in $[9,8,10]$, which are in much better agreement with the tokamak and RFP databases. They describe the density limit as a radiative one, and therefore include naturally a clear explicit dependence on $P$. The theory and good agreement with experiments extends to the stellarator $[9,11,12,10]$, which provides a unified view of density limits in magnetic confinement.

A large part of the radiative density limit comes from the radiation of impurities $[9,8,10]$. Therefore, one may try to push this limit toward higher values by increasing the plasma purity. The latter is governed by plasma-wall interaction. A lot is known 
about this interaction, but it has not yet been considered self-consistently with the whole plasma. This is the purpose of this paper, which introduces the concept of selforganization of the plasma-wall system by simple zero and one-dimensional models. The basic idea of the theory is the existence of a time delay in the feedback loop relating radiation and impurity production on divertor plates. It yields an iterative equation for radiation whose fixed point corresponds to the plasma-wall equilibrium. This equilibrium can be unstable for a ratio of total radiated power to total input power as low as $1 / 2$.

Most of this paper is devoted to the tokamak, but the stellarator and the reversed field pinch are considered too. Section 2 deals with the sputtering of divertor/limiter targets due to plasma ions. Section 3 deals with the density and radiation of impurities originating from these targets. Section 4 introduces a 0-dimensional description of plasma-wall self-organization (PWSO), and its extension to a 1-dimensional one is provided in Appendix $\mathrm{C}$ in order not to interrupt the flow of the article. Section 5 provides elements of experimental support to the theory. Section 6 provides a discussion of the results. Section 7 discusses start-up in the stellarator and the tokamak. Section 8 makes experimental proposals in order to increase the density limit in the tokamak, the stellarator, and the RFP by an appropriate tailoring of gas injection and electron cyclotron resonance heating (ECRH). Then comes the conclusion.

\section{Sputtering of the targets due to plasma ions}

We consider the targets of a divertor or the edges of a limiter connected to the plasma by a SOL with a temperature $T_{t}$ at these boundaries, called targets in the following. In front of a target, there is a Debye sheath of height $\gamma T_{t}$, with $\gamma$ a few units (see equation (25.46) of [13], $\gamma \simeq 7$ for hydrogen, vanishing secondary electron emission, and $T_{t}$ in $\mathrm{eV})$. Therefore, a plasma ion with a velocity $v$ at the sheath entrance impinges it with an energy $E=c v^{2}+\gamma T_{t}$, where $c=M / 2$, with $M$ the ion mass. Let $Y(E)$ be the yield function for the compound chemical and physical sputterings of the target by an ion with energy $E$. Interpolating functions for $Y(E)$ at normal incidence are provided in [14] for physical sputtering, and figure 3.8 of [13] provides this yield for the chemical sputtering of carbon. In reality, the yields should be corrected for the slanting and roughness of the targets implying a non normal incidence; also for self-sputtering. The number of sputtered atoms per unit time on the two divertor targets is

$$
s=\frac{P_{t}}{T_{t}} I\left(T_{t}\right)
$$

with $P_{t}$ the power reaching the target and

$$
I\left(T_{t}\right)=\sqrt{\frac{c}{\pi T_{t}}} \int_{0}^{\infty} Y\left(c v^{2}+\gamma T_{t}\right) \exp \frac{-c v^{2}}{T_{t}} \mathrm{~d} v,
$$

where the lower boundary in the integral takes into account the fact that only velocities directed toward the targets are active.

For carbon, according to section 3.3.2 of [13], for incident energies $E_{0}$ "of hundreds $\mathrm{eV}$ chemical sputtering yields are comparable to or larger than physical sputtering 
yields". Furthermore, the "strong dependence on substrate temperature is one of the key signatures of chemical sputtering, [... with a peak yield occurring at a substrate temperature which depends on $E_{0}$, but generally lying in the region of $700 \mathrm{~K}$ ". The dependence of $Y$ on $E_{0}$ is weak according to figure 3.8 of [13]. Typically, the substrate temperature grows with the incident heat flux, and therefore with $T_{t}$. So does $I\left(T_{t}\right)$. For simplicity, we assume $I\left(T_{t}\right) / T_{t}$ almost constant in the following for carbon.

Since the density limit corresponds to rather high collisionality, it is natural for its description to assume parallel heat conduction to dominate over parallel heat convection (see sections 4.8 to 4.11 of [13]). Problem 4.12 of [13] yields a table showing that in this regime, for a heat flux going from $10^{8}$ to $10^{9} \mathrm{~W} / \mathrm{m}^{2}, T_{t}$ goes from 27 to $317 \mathrm{eV}$. Appendix A shows that for physical sputtering of tungsten by deuterons, $I\left(T_{t}\right) / T_{t}$ is almost constant for the part of this interval above $100 \mathrm{eV}$. The precise meaning of "almost" is discussed at the end of section 4.1 .

\section{Density and radiation of target impurities}

We introduce a simple model of thermonuclear plasma. It deals with a magnetized cylindrical plasma of length $L$ with axial and azimuthal symmetry bounded by a cylindrical wall of radius $a$ with the same axis. There is a scrape-off layer (SOL) of width $\lambda_{S O L} \ll a$ in contact with the edge targets of a limiter or the targets of a divertor, which sets the last closed flux surface (LCFS) at $a-\lambda_{S O L}$. We call $T_{t}$ the plasma temperature at the targets. It is in units of energy as all other temperatures in this paper. We consider the SOL to provide the main loss of ions reaching the LCFS. Indeed, because of the ambipolar drift it induces [15], the particle collection by a localized limiter is not efficient.

We assume that some fraction $f$ of the sputtered atoms reaches the main plasma and is ionized at a distance $\lambda_{i}$ inside the LCFS. Keeping in mind the cylindrical description as a background reference, we further restrict it to a slab model, and we use the Engelhardt and Feneberg one, as described in [13], to compute the density of impurities. This yields $n_{i p}=\frac{f s \lambda}{2 \pi a L D_{\perp}}$ (equation (6.6) of [13]), where $D_{\perp}$ is the perpendicular diffusion coefficient of target impurities, and

$$
\lambda=\lambda_{i}+\lambda_{S O L}
$$

Combined with equation (1), this yields

$$
n_{i p}=\frac{f \lambda P_{t}}{2 \pi a L D_{\perp} T_{t}} I\left(T_{t}\right)
$$

Then, the total radiation due to target impurities is

$$
A=\frac{f \lambda P_{t}}{a D_{\perp} T_{t}} I\left(T_{t}\right) \int_{0}^{a} r n(r) \operatorname{Rad}[T(r)] \mathrm{d} r
$$

where $\operatorname{Rad}(\mathrm{T})$ is the target impurity radiation rate coefficient, and $n(r)$ and $T(r)$ are respectively the density and temperature profiles. 


\section{Zero dimensional description}

When describing a complex system, there is always a trade-off between economy of means and explanatory power. In this section, we first chose the economy of means and introduce a 0-dimensional description of PWSO. Appendix C provides a 1-dimensional one, which makes more explicit the physical mechanisms underlying the 0-dimensional description. It couples an impurity transport equation and a heat transport equation, which are respectively of the kind used in the Engelhardt and Feneberg, and in the Zanca et al. models.

\subsection{Simplest version}

Let us assume, for a given total injected power $P$, the existence of a stationary state for the plasma-wall system with density and temperature profiles $n_{e q}(r)$ and $T_{e q}(r)$. In the following, we will consider states of the system close to this equilibrium. Then, in (5) $T_{t}$ depends on $P_{t}$ in various ways depending on the SOL regime of interest, as will be discussed later. Therefore, we consider $A$ as a function of $P_{t}$ only, $A\left(P_{t}\right)$.

Let $R$ be the total radiated power at time $t$. Then the power landing on the targets is $P-R$. This produces sputtered atoms, which need a finite time of flight to reach the ionization position $a-\lambda$. Then they start radiating after a diffusion/propagation time

for impurities. This radiation is felt by the targets after a diffusion/propagation time for heat. The sum of these three times defines a delay $\tau_{\text {delay }}$ for setting a new value $R_{+}$ of $R$.

The simplest version of our iterative model for radiation considers $R$ as due to $A$ only. Then, setting $P_{t}=P-R$ yields

$$
R_{+}=A(P-R),
$$

We consider $R$ as close to $R_{e q}$ the equilibrium value of $R$, which corresponds to $R_{+}=R$ in this equation.

We further assume $I\left(T_{t}\right) / T_{t}$ to be almost constant, as was shown to be reasonable at the end of section 2 for tungsten at temperatures in the range 100-300 eV, and for carbon. Therefore, we first consider the case where

$$
\alpha=\frac{A\left(P_{t}\right)}{P_{t}},
$$

is a constant. Then the iterative equation (6) becomes the linear relation

$$
R_{+}=\alpha \times(P-R) .
$$

$R_{e q}$ is the fixed point of this iterative equation. Equation (8) implies

$$
\alpha=\frac{R_{e q}}{P-R_{e q}}=\frac{\mu}{1-\mu},
$$

where $\mu=R_{e q} / P$. If $\alpha>1$, the fixed point $R_{e q}$ of equation (8) is unstable, since $R_{+}-R_{e q}=-\alpha\left(R-R_{e q}\right)$. Equation (5), shows that for $I\left(T_{t}\right) / T_{t}$ almost constant, $\alpha$ is a growing function of the density in the radiating region. Therefore, the just found instability threshold corresponds to a density limit. 
Equation (5) contains several parameters whose value is not well known in experiments. However we now give two estimates showing that $\alpha$ takes values in the right ballpark. First, for tungsten by setting in (5) $f=1, \lambda=10^{-2} \mathrm{~m}, D_{\perp}=1 \mathrm{~m}^{2} / \mathrm{s}$, $T_{t}=100 \mathrm{eV}$, the ratio of $I\left(T_{t}\right)$ to its maximum value $Y_{\max }, I\left(T_{t}\right) / 310^{-3}=\kappa$ where $\kappa$ is a number smaller than 1 , and the integral is computed by assuming $n(r)=10^{20} \mathrm{~m}^{-3}$, and $\operatorname{Rad}[T(r)]=10^{-28} \mathrm{Wm}^{3}$ over a length $l=10^{-2} \mathrm{~m}$ about $a$ and 0 outside. This yields $\alpha \simeq 210^{2} \kappa$, which provides $\alpha=1$ for $\kappa=0.510^{-2}$. This ratio of $I\left(T_{t}\right)$ to its maximum value necessary to reach a density limit at $n(r)=10^{20} \mathrm{~m}^{-3}$, is a reasonable order of magnitude estimate in view of the large uncertainty on the above parameters. The value of $Y_{\max }$ used in this estimate are provided at the end of Appendix A, and those for $\operatorname{Rad}[T(r)]$ are in Appendix A of [8].

Second for carbon. We keep the same numerical values, except $\operatorname{Rad}[T(r)]=$ $10^{-32} \mathrm{Wm}^{3}$ and $I\left(T_{t}\right)=310^{-2}$. This yields $\alpha \simeq 0.2$. From this level, $\alpha=1$ can readily be obtained by reasonable variations of the parameters in (5) used above for tungsten, in particular $l$ and $\lambda$. Therefore, $\alpha=1$, as provided by (5), is a reasonable threshold for present density limits with both carbon and tungsten targets. The value of $Y_{\max }$ used in this estimate are provided in figure 3.8 of [13], and those for $\operatorname{Rad}[T(r)]$ are in Appendix A of [8].

$\alpha$ is a growing function of $\mu$, and becomes larger than 1 if $\mu>1 / 2$. Therefore, the simplest version of our iterative model for radiation opens the prospect of a radiative density limit occurring for a fraction of radiated power as low as $1 / 2$. After the outcome of [9] recalled in the introduction, this is another prediction of a radiative density limit without necessarily a very high fraction of radiated power.

In reality, the above assumption of constant $I\left(T_{t}\right) / T_{t}$ and $\alpha$ means the dependence of $A\left(P_{t}\right)$ on $P_{t}$ is dominated by the explicit coefficient $P_{t}$ in (5). Appendix B shows that if $A\left(P_{t}\right)$ departs from a linear dependence on $P_{t}$, but keeps being a growing function of it, the above fixed point and stability analysis can still be performed. This is important for tungsten at low $T_{t}$, where $I\left(T_{t}\right) / T_{t}$ varies a lot, as shown at the end of Appendix Appendix A.

If there is detachment, there is a strong neutral radiation in the SOL, and $T_{t}$ decreases (see figure 5 a of [16], quoted as figure 29 in [17]). This implies a vanishing $I\left(T_{t}\right) / T_{t}$ in $A\left(P_{t}\right)$ if physical sputtering dominates, since $I\left(T_{t}\right)$ decreases very fast at low $T_{t}$. This pushes the radiative density limit to very high values, in particular for tungsten. Vanishing sputtering at low $T_{t}$ is more visible in the one-dimensional description of Appendix $\mathrm{C}$, since it takes into account the power radiated in the SOL, which diminishes both $P_{t}$ and $T_{t}$ when approaching detachment.

Therefore, our simplest model predicts a very high density limit for targets where physical sputtering dominates, if the system is initially set at a high enough density. Therefore, the simplest model of PWSO comes with two basins for this organization: the usual one with a density limit, and a new one with density freedom. 


\subsection{Other contributions}

In reality, there is a lot more radiation than that due to target impurities, especially with impurity seeding. In particular, neutral particles are strong contributors. They are highly localized both radially (in the SOL) and poloidally (in the limiter/divertor region). Therefore the modelling of their radiation is more problematic. Reference [18] introduces the ratio $Q_{*}$ between the powers radiated by neutrals and impurities in the peripheral region, and shows it to be between 1 and 5, according to experimental measurements. This ratio can be used to account for the radiation of neutral particles by adding a factor $1+Q_{*}$ to the previous expression for $A$, which does not modify qualitatively the above analysis, and keeps the $\mu=1 / 2$ threshold.

Instead of a radiation of neutrals proportional to that of impurities, one can also consider neutrals whose radiation does not depend on $P-R$. This can be accommodated by adding a constant term $R_{0}$ in the right hand side of equations $(6,8)$. The previous threshold in $\mu$ becomes $\mu>\left(1+R_{0} / P\right) / 2$. It increases with $R_{0}$, since the weight of the feedback contribution is decreasing in equations $(6,8)$.

For heavy impurities, both neoclassical and turbulent pinch velocities can lead to their accumulation in the plasma center [19]. If this accumulation is slow with respect to $\tau_{\text {delay }}$, the corresponding radiation can also be accommodated by adding a constant term $R_{a c c}$ in the right-hand-side of equations $(6,8)$. The previous threshold in $\mu$ becomes $\mu>\left(1+R_{a c c} / P\right) / 2$. The same technique can be used if a part of the impurities does not originate from the targets.

The last two paragraphs describe cases where the density limit threshold sets in for $\mu>1 / 2$. Experimentally, most experiments correspond to this case.

In the regime of plasma detachment at high density with a low target temperature, one might reach a regime with $\mu=1$ where the whole input power is radiated in the SOL by neutrals. Then, neutral radiation would be produced very close to the targets where recycling is occurring. This would make irrelevant the finite distance and delay, which are essential in the iterative model, and no unstable feedback would be present. The same might exist with impurity seeding, but the above calculation of sputtering should be revised accordingly. So the existence of other contributions to radiation than the one due to target impurities does not preclude that of a basin of density freedom if their radiation level stays low.

\subsection{Helical systems}

In [9], [11], and [12], it was found that a cylindrical description of the stellarator yields a good description of its density limit as a radiative one. References [9] and [10] show the same property holds for the reversed field pinch (RFP). Therefore, it is natural to extend the 0D and 1D models of PWSO to these two devices as well. 


\section{Experimental support}

According to (8), when $\alpha>1, R-R_{e q}$ has an exponential growth with a growth rate $\tau_{\text {delay }}^{-1} \ln (\alpha)$ and with alternating positive and negative values. Some experimental results suggest the existence of such an oscillating instability.

In particular figure 2 of [20], which displays large oscillations of the radiation for the JET belt limiter in the non evaporated beryllium case, with a time scale of 1 s typically. Also in figure 13 of [21], which corresponds to a similar experiment and displays two alternations of $R$, followed by a decrease of $P$. Furthermore, at page 527 of [5], which deals with the density limit in W7-AS, it is stated: "Whenever a rather strong collapse is observed, pictures of tangentially viewing video cameras show, near the inner plasma edge, the sudden appearance of a bright area which changes with time. Correlated with this observation are discontinuous reductions of the $H_{\alpha}$ signals from the limiters and a sudden rise in the C III radiation, indicating a transition to very low temperatures".

To the contrary, the avoidance of the unstable regime might explain why disruptions can be avoided in the JET-ILW [2]. This avoidance would come from plasma detachment and a consequent vanishing of sputtering on the targets. Reference [20] reports : "in the [beryllium] evaporated case the radiation did not normally symmetrise and persisted at $100 \%$ radiation, usually without disrupting". The reason for this can be understood in the frame of our theory, since at the density limit, chlorine produces the whole radiation, which quenches $A\left(P_{t}\right)$.

Regimes with a subdominant $A\left(P_{t}\right)$ can be inferred for W7-AS in [22]. There is a collapse occurring when total radiation and input power are balanced. In these cases, the plasma density and radiation profiles were peaked, with a dominant core radiation from higher $\mathrm{Z}$ elements.

\section{Discussion}

We found that the radiative density limit due to PWSO can be reached for a ratio of total radiated power to total input power as low as $1 / 2$. Such a possibility was already found in [9], which used a prescribed amount of radiators. This is important, since the fact that the density limit is not always associated with a very high radiated fraction was used as an argument against the radiative origin of this limit (see section III.E.1 of [23]). The density limit in [9] corresponds to a threshold for the occurrence of non monotonic temperature profiles. Such a threshold had been found previously in [24] as that where Bessel-like oscillations of the temperature profile become unstable. Therefore, there are at least two different radiative density limits. One due to the feedback of plasma-wall interaction on impurity radiation, corresponding to PWSO, and the other one due to the instability of monotonic radial profiles in the simple power balance model (PBM) of $[9,8,10]$.

For the disruptive limit of the tokamak $[9,8,10]$ and for the radiation collapse for the stellarator $[9,11,12,10]$, the PBM predictions are in good agreement with experimental measurements, with a small scatter of experimental points about the theoretical curves. 
A precise PWSO threshold is difficult to estimate from its definitions (7) and (C.13). However, the fact that both PBM and PWSO models give a threshold in $R / P$ between $1 / 2$ and 1 suggests that their density thresholds are close, and is in agreement with the fact that $\alpha=1$, as provided by (5), was found to be a reasonable threshold for present density limits with both carbon and tungsten targets in section 4.1.

In $[9,10]$, the PBM estimate for the RFX-mod RFP is an upper bound of the plasma densities of a large set of discharges. The experimental points become scarce in the vicinity of this bound. This suggests the PWSO threshold might be reached in this device with carbon walls. This is possible, since section 4.1 found that $\alpha=1$ can be reached with a reasonable choice of parameters for carbon impurities. Even more, if the sublimation of carbon under strong irradiation is taken into account. Indeed, in the multiple helicity state of the RFP, there is a strongly localized interaction of the plasma with the wall.

As indicated in section 5, the PWSO threshold might have been reached in devices with metallic walls in the past. Indeed, section 4.1 found that $\alpha \simeq 210^{2} I\left(T_{t}\right) / Y_{\max }$ with a reasonable choice of parameters for tungsten, which makes $\alpha=1$ very easy to reach. This motivated the passage to more compliant carbon walls.

If its threshold is not reached, PWSO defines the plasma-wall equilibrium and its corresponding amount of target impurities. Then, the self-consistency of the impurity and heat transport equations of Appendix $\mathrm{C}$ can be neglected and a thermal equilibrium can be computed as in $[9,8,10]$, or the stability analysis of this equilibrium can be performed as in [24]. If the plasma-wall system is in the basin of density freedom defined by PWSO, then this freedom is kept by the PBM, since the radiation of target impurities vanishes. Indeed, scaling (20) of [8] for the density limit for the tokamak incorporates a factor $Z_{e f f}^{4 / 9}\left[f_{0}(\%)+Z_{e f f}-Z_{i}\right]^{-5 / 9}$, which becomes large when the effective charge $Z_{\text {eff }}$ comes close to $Z_{i}$, the main ion one $\left(f_{0}(\%)\right.$ is the percentage of neutral concentration).

We now address the role of thermal transport in PBM and PWSO. In the case of PBM, its effect is included in the global quantity of the scalings. For the pinches, it is only present in a factor $\left(\tau_{E}\right)^{-1 / 9}$, in a form factor depending on the conductivity radial profile, and in the loop voltage $V_{\text {loop }}$, which is present by the term $P /\left(V_{\text {loop }} I_{p}\right)^{0.5}[8]$. Anyway, $V_{\text {loop }}$ has limited variations for the tokamak with respect to those of $I_{p}$ and $P$. For the stellarator, the effective diffusivity has a larger exponent, but the use of the International Stellarator scaling 95, or of the scaling law for the effective thermal diffusivity established in the low temperature region of LHD yields scaling laws for the density limit, which are in good agreement with experiments [9]. For PWSO, the effective diffusivity is present in (C.13) by a small effect in the definition of $\lambda_{\text {rad }}$ and indirectly through $T_{t}$.

We end this discussion by quoting approaches to the density limit relying on different physics than those used in the PBM and PWSO. Two works appeal to the excess of radiation of thermal structures produced by magnetic islands for the tokamak [25] and the RFP [26]. This does not preclude the density freedom predicted by PWSO, since this radiation is bound to vanish if the impurity density does. Another approach shows the existence of an edge shear layer collapse for a density scaling like plasma 
current in the tokamak $[27,28]$. An experiment in HL-2A showed accordingly that when the density increases toward Greenwald estimate, the turbulent particle transport increases drastically [29]. Therefore, as stated in [9], several mechanisms might be occurring at the density limit. More work is necessary to make a detailed comparison of the predictions of theoretical models with experiments. However, as yet only the approach of $[9,8,10]$ has provided scalings for the density limit in good agreement with a broad database for the tokamak, the stellarator, and the RFP. This gives confidence in the leading role of radiation in the density limit and in the importance of PWSO.

\section{Start-up}

The simplest model of PWSO in section 4.1 took only into account the feedback on radiation due to target impurities. Then, a very high density limit can be predicted, if the system is initially set at a high enough density. In subsection 4.2 , we considered other sources whose radiation does not depend on $P-R$. They may impose a finite density limit, but it may be a high one. Furthermore, a strong radiation in the SOL can help reaching low temperatures on the targets, and thus their low sputtering. Therefore, a high enough initial density can be maintained in the frame of the above PWSO. By initial density, we mean the one reached at the end of start-up. The maximum possible density at this moment is bounded by the amount of impurities, which are produced during start-up. The next subsections address the issue of how to decrease this amount.

\subsection{Stellarator start-up}

In stellarators, plasma breakdown is generally performed with waves, often ECRH ones. In W7-X, after an initial phase where ECRH start-up was performed at low neutral density and low ECRH power, they went to high values of these quantities reaching plasma densities above $10^{20} \mathrm{~m}^{-3}$ with almost fully absorbed $6 \mathrm{MW}$ ECRH heating power (see section 3 of [30]). This came with several benefits: (i) an efficient heating of ions by the electrons, leading to almost thermal equilibration; (ii) a fully detached divertor and a drop of the power load on the divertor targets. These high densities were reached thanks to boronization. Then, "low-Z impurities in the plasma were significantly reduced, resulting in a profound effect on increasing the density limit (at a given heating power). The critical density increased by about a factor of 3 , corresponding to a decrease in the low-Z impurity concentration by factors between 5 and 10 . At a heating power of $5 \mathrm{MW}$, this meant that the line-averaged densities of $10^{20} \mathrm{~m}^{-3}$ became accessible" (bottom of page 7 of [31]). It is remarkable that in $100 \mathrm{~ms}$ of $2 \mathrm{MW}$ ECRH injection, a density of $210^{19} \mathrm{~m}^{-3}$ (an order of magnitude higher than in tokamak breakdown) is reached with a single gas puff, right before injecting waves; then $10^{20} \mathrm{~m}^{-3}$ is reached by increasing ECRH power to $4 \mathrm{MW}$ without any further gas puffing [32]. 


\subsection{Traditional ohmic start-up of the tokamak}

In order to ease plasma breakdown, the traditional ohmic start-up of the tokamak is usually performed at a density much lower than the one targeted during the current plateau. Close to the wall, there are hot plasma zones directly linked to the magnetic separatrices (see fig. 10 of [33]). Because of the low density, the plasma interacts strongly with the walls, by means of ExB drifts and secondarily of open magnetic field lines [33]. This direct interaction with the walls lasts till the formation of closed magnetic surfaces and produces a lot of impurities, in particular during the burn-through phase. "In this phase, low-Z impurities, usually originating from the walls surrounding the plasma column, radiate and can limit the temperature and the current ramp-rate so that the discharge fails" [34].

\subsection{Two opposite regimes of start-up for the tokamak}

If the density during start-up is on the low side, there is a vicious circle: (i) there is no screening of the targets by neutrals and a high temperature favorable to sputtering; (ii) no plasma detachment from the walls can occur, and even no help to decrease the heat and particle loads leading to sputtering. Therefore, a high level of impurities is maintained, leading to the usual density limit at flat top. A high level of impurities is even more maintained, whenever they accumulate in the plasma center. This scenario corresponds to the traditional ohmic start-up of tokamaks: the maximum possible density, which can be reached by later gas puffing or pellet injection at the end of the start-up, is bounded by the amount of impurities, which are produced during this initial period.

To the contrary, a high density during start-up would trigger a virtuous circle: with a high plasma density, the walls would be screened by neutrals and temperature might be kept low during current ramp-up, up to the formation of closed magnetic surfaces. This would produce few impurities, maintaining a high density limit, and would ease current penetration, enabling a faster current ramp-up. When closed magnetic surfaces would form, heat and particle loads leading to sputtering would be low, and even plasma detachment from walls and targets could occur. Again, this would produce few impurities, keeping a high density limit. We show below that a strongly ECRH assisted start-up might enable reaching this high density scenario.

The above vicious and virtuous circles are two basins of attraction of a second aspect of PWSO. Unfortunately, usual tokamak operation is trapped into a bad basin of PWSO for this second aspect. As yet, PWSO leads to better basins of operation in stellarators, with a higher density limit than in pinches [9].

We would like to stress that the above vicious circle is independent of the particle confinement time. Indeed, start-up determines an initial density limit. If this limit is on the low side, no plasma detachment from the walls can occur, even no help to decrease

the heat and particle loads that lead to sputtering. So, a high level of impurities is maintained, which leads to the usual density limit estimate. The high level of impurities is even more maintained, whenever they accumulate in the plasma center. So the issue 
is not the memory of the plasma, but that of the plasma-wall system: the trapping of operation into a bad basin of PWSO.

The hope that a strongly ECRH assisted start-up described below might enable reaching the above high density scenario is especially strong for walls with high$\mathrm{Z}$ materials. Indeed, the lower sputtering yield for such materials at low plasma temperature make them less a problem at start-up. Reference [34] brings some support to this: "Also, the radiated power at the time of burn-through is a steep function of density for the carbon wall but weakly dependent on density for the ILW. The latter point is likely due to the much reduced radiative cooling from Be compared to $\mathrm{C}[. .$.$] as$ well as chemical sputtering of $\mathrm{C}$ from the graphite wall. Furthermore, on JET with the ILW, there were no failures of the start-up during the burn-through phase or failures due to deconditioning events, such as disruptions or excessive gas puffing on the previous shot, unlike with the graphite wall".

\section{Experimental proposals}

Since the density limit is due to impurities, one may try to push this limit toward higher values by increasing the plasma purity. For tokamaks, the passage to tungsten walls and producing divertor detachment at high densities is already an important step in this respect [2]. Improving the start-up of the discharge might bring a new strong benefit. This is suggested by the just described higher density limit in stellarators when the start-up is performed by using high power ECRH [30, 31]. This higher limit might be not intrinsic to the stellarator, but only to its mode of plasma breakdown: the massive use of ECRH with high neutral density.

In the tokamak, till now, for assisting ohmic start-up by easing breakdown and increasing the power available to burn through impurity radiation, ECRH was used at a low level, and with densities an order of magnitude lower than those reached during the flat top. The general motivation for this procedure was the difficulty for fully superconducting tokamaks to produce the high loop voltages necessary for breakdown. However, a more strongly ECRH assisted ohmic start-up might alleviate the unpleasant constraints of ohmic start-up (low density, slow ramp up) leading to strong impurity production. Indeed, fortunately, ECRH assisted start-up was not acrobatic at all. Therefore, there are margins for using higher powers with higher initial neutral densities, possibly similar to those in the recent experiments in W7-X quoted above. This could be checked by increasing progressively these two quantities in tokamaks equipped with a high ECRH power. Several tokamaks have an available ECRH power much higher than that necessary for an assisted ohmic start-up. For instance, TCV has an installed ECRH power of 4.5 MW, while only 0.4 MW were used to start the plasma and sustain the plasma current (section 3.3 of [35]). This tokamak and other middle size ones might try a higher power assisted breakdown scenario, without suffering from bad consequences of possible disruptions during the experiments. It is worth noting that the results on the range of possible prefill pressures observed on several experiments show that it may be increased by high power EC assist by a factor of two or three, without attempting 
any optimization [36].

In contrast to the stellarator, closed magnetic surfaces are not present during a large part of the plasma start-up in a tokamak. However, in an ECRH assisted ohmic start-up, the walls are more shielded than in an ohmic one, since the plasma is produced far from the walls close to the EC resonant layer (in reality at the upper hybrid layer $[37,38])$. This is quite visible by comparing figures 2 and 3 of [39]; the discharge limits on the inner wall only after the formation of closed magnetic surfaces.

A higher neutral density should decrease plasma temperature and increase the shielding of the wall from the plasma. This should decrease the production of impurities before the formation of closed magnetic surfaces. However, for machines with carbon walls, it would be advisable to perform boronization or a similar wall conditioning to avoid chemical sputtering. As quoted above, this was crucial for the above quoted good W7-X results, even though good magnetic flux surfaces are present from the outset in the machine.

In tokamaks able to perform an ECRH assisted ohmic start-up, it would be interesting to compare the $Z_{\text {eff }}$ in this case and in the ohmic one. An improvement might already be present. In tokamaks having an available ECRH power higher than that necessary for an assisted ohmic start-up, one could try an ECRH assisted breakdown with a density larger than the one used till now, since there might be some margin in density with respect to the cutoff one. It would then be interesting to check whether the $Z_{\text {eff }}$ at flat-top decreases, after increasing the density at the end of the ECRH assisted phase. Even without having a density limit lower than that of cutoff, it would be interesting to get the largest possible density after ECRH breakdown. This might provide a cleaner plasma during flat-top, after the subsequent gas puffing or pellet injection, and might push the density limit above the usual one. Even without reaching density freedom, higher density limits might be reached.

Other benefits might result from this new scenario: (i) saving more volt-seconds than in the traditional ECRH assisted breakdown, since the current plateau density would be reached right away; (ii) reaching divertor detachment earlier, in particular because one would avoid the hollow neutral density profile coming with gas puffing [2]; (iii) fulfilling more easily Lawson criterion. Other benefits might come like an easier transition to $\mathrm{H}$ mode, taming some instabilities, a less crucial divertor design, less disruptions, etc... Fulfilling more easily Lawson criterion is the most exciting benefit. Indeed, this might lead to more margins for ITER and fusion reactors smaller than expected presently. A first version of this experimental proposal is already available in [40]. Indeed, it could be motivated without the full derivation of the analytical part of this paper.

As stated in [34], "in general, the start-up phase of discharges gets attention only when there is a failure". Start-up is poorly diagnosed, it is largely empirical for each device, and few papers are devoted to it. The above discussion suggests that tokamak flat-top operation would benefit a lot from a thorough study of this phase.

As yet, PWSO leads to better basins of operation in stellarators, with a higher density limit than in pinches [9]. However, as yet there was no attempt to improve upon 
this by an appropriate tailoring of gas injection and ECRH power input. Margins are available for this, and even more when the full $10 \mathrm{MW}$ ECRH power will be available in W7-X. A hint is that, on top of the basin described in subsection 7.1, there is even a better one: the highest performance is achieved in W7-X with pellet fueling [30]. This fueling sets operation in a basin disconnected from the traditional one where fueling is coupled to the edge neutral density.

In the RFP, during breakdown the magnetic field is much lower than during the flat-top, and therefore the electron cyclotron frequency is low with respect to that in tokamaks. However, ECRH assisted start-up might work as well. As for the tokamak, separating breakup and current rise might enlarge the operation space, and provide cleaner plasmas.

\section{Conclusion}

This paper introduces the concept of plasma-wall self-organization (PWSO) in magnetic fusion plasmas, and derives a theory describing it. Its basic idea is that there is a time delay in the feedback loop relating radiation and impurity production on divertor plates. Both a zero and a one-dimensional description of PWSO are provided. Both lead to a delay equation whose simplest expression is $R_{+}=\alpha \times(P-R)$, where $P$ is the total input power in the plasma, $R$ is the total radiated power, and $R_{+}$is its delayed value. This makes the plasma-wall system unstable for $\alpha>1$. Since $\alpha$ is proportional to the density below detachment, this threshold defines a density limit. It can be reached for a ratio of total radiated power to total input power as low as $1 / 2$. If this threshold is not reached, the self-consistency of impurity and heat transport can be neglected, a thermal equilibrium can be computed, and its stability analyzed in the frame of the simple power balance model.

When detachment develops, the plasma temperature at the targets decreases, which makes $\alpha$ to vanish. This pushes the radiative density limit to very high values when physical sputtering dominates, in particular for tungsten. Therefore, PWSO comes with two basins for this organization: the usual one with a density limit, and a new one with density freedom. If the plasma-wall system is in the basin of density freedom defined by PWSO, then this freedom is kept by the simple power balance model. A lot is left to provide more realistic descriptions of this organization. This will involve computer calculations of various scales, up to a numerical tokamak.

When $\alpha>1$, the deviation of radiation to its equilibrium value starts an exponential

growth with alternating positive and negative values. Some experimental results suggest the existence of such an oscillating instability.

When physical sputtering dominates, a very high density limit can be predicted, if the plasma is set at a high enough density at the end of start-up. The maximum possible density at the end of start-up is bounded by the amount of impurities, which are produced during this period. This amount is high for the traditional ohmic start-up of the tokamak, which is performed at low density. This sets the system in a vicious circle where a low density limit prevents reaching the high densities enabling detachment. To 
the contrary, a high density during start-up would trigger a virtuous circle enabling it. These vicious and virtuous circles are two basins of attraction of a second aspect of PWSO.

Unfortunately, usual tokamak operation is trapped into the bad basin of PWSO. As yet, PWSO leads to better basins of operation in stellarators, with a higher density limit than in pinches. This is likely due to their ECRH start-up, and suggests that a strongly ECRH assisted ohmic start-up might enable reaching the high-density scenario, especially for targets with high-Z materials. This would bring several benefits. Especially fulfilling more easily Lawson criterion, which might bring more margins for ITER and lead to smaller fusion reactors than expected presently. A strongly ECRH assisted ohmic start-up might be beneficial for the RFP as well. Stellarators might take advantage of improving their present start-up scenarios toward higher initial densities.

The crucial role of the start-up phase in tokamaks suggests to study it more thoroughly. Indeed, flat top fusion plasmas are like human beings: they are strongly defined by genetics and education. In order to better understand them, their baby and teenager states should be studied...

Evolution eased the interaction of birds and of cetaceans with their fluid environment by the development of feathers and of an intelligent skin. On the technological side, a similar evolution occurred with the development of ultra-fast and silent torpedoes bathed in supercavitation bubbles. More pacific, though unconscious, a similar evolution is in progress in the tokamak. Indeed, motivated by the management of tritium inventory, the dreaded, but finally marvelous passage to tungsten occurred. It enabled the production of detached plasmas at high density because they are cleaner. More studies about start-up might suggest new steps in establishing peaceful relations between the walls of magnetic fusion devices and their thermonuclear plasma environment.

If the present density limit could be overcome in present medium size machines, this would open the prospect of more margins for ITER and of smaller size reactors. Decreasing the size would also decrease the wall load challenge. This in turn would decrease the need for impurity seeding in reactors, opening the prospect of another virtuous circle. While it would be far fetched to take for granted the existence of such a circle, it is worth thinking about its possibility, in view of the impressive tokamak DEMO wall load challenge [41]. The above ideas can be checked in medium size machines which do not suffer from disruptions, and whose experimental handling is much simpler than in larger devices. Taming fluid turbulence looked like an overwhelming task, but the development of ultra-fast and silent torpedoes bathed in supercavitation bubbles brought a large progress toward its cure. Similar good news might be ahead for magnetic fusion.

\section{Acknowledgments}

C. Arnas, L. Carraro, M. E. Puiatti, M. Valisa, and M. Wischmeier are thanked for information about plasma-wall interaction and impurity radiation. X. Bai, J. Bucalossi, S. Coda, H. de Oliveira, A. Fasoli, J. Graves, K. Nagasaki, Y. Peysson, H. Reimerdes, B. 


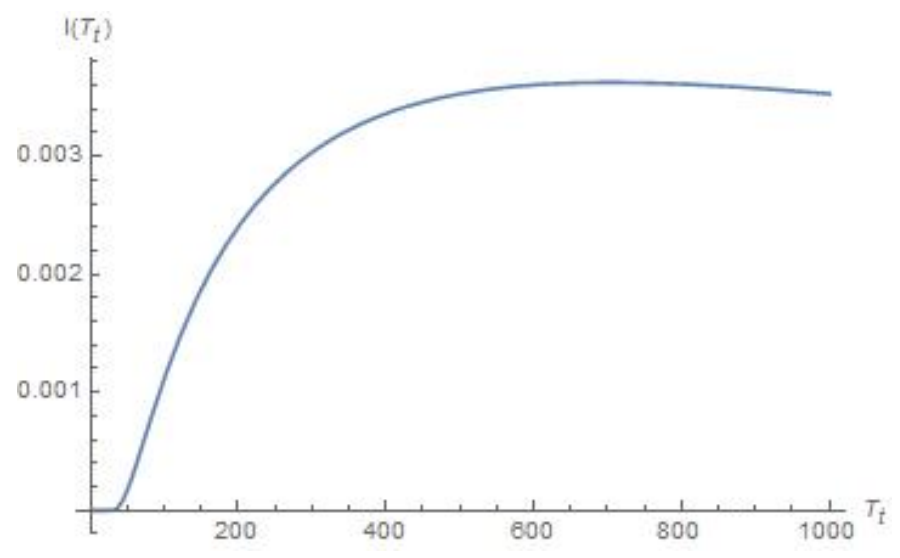

Figure A1. $I\left(T_{t}\right)$ for physical sputtering of tungsten by deuterons.

Shukla, and J. Stober are thanked for their help in discussing the experimental proposals. T. Klinger and H. Laqua are thanked for unpublished information about W7-X start-up. S. Cappello is thanked for an improvement in the large scale description of the theory. One of us (DFE) is grateful to the 2019 Festival de Théorie for the interaction with L. Howlett who pointed out to him Stacey's work [24].

\section{Appendix A. Physical sputtering}

In order to get a simple estimate of $I(T)$ in (2), we approximate $Y(E)$ by square functions equal to $Y_{\max }$ for $E_{m}<E<E_{M}$ and zero outside. Let $x=\sqrt{c} v$, $x_{m}=\sqrt{\sup \left(E_{m}-\gamma T_{t}, 0\right)}$, and $x_{M}=\sqrt{\sup \left(E_{M}-\gamma T_{t}, 0\right)}$. Then

$$
I\left(T_{t}\right)=\frac{Y_{\max }}{\sqrt{\pi T_{t}}} \int_{x_{m}}^{x_{M}} \exp \frac{-x^{2}}{T_{t}} \mathrm{~d} x .
$$

$I\left(T_{t}\right)$ vanishes for $T_{t}=0$, then grows and reaches a maximum almost equal to $Y_{\max }$ for $T_{t}=T_{\text {sputtmax }} \equiv E_{m} / \gamma$, since typically $E_{m} \ll E_{M}$ (see [14]); then it decreases to vanish for $T_{t}=E_{M} / \gamma$.

Figure (A1) and (A2) display respectively $I\left(T_{t}\right)$ and $I\left(T_{t}\right) / T_{t}$ for physical sputtering of tungsten by deuterons. The calculation uses data of [14], with $Y(E)$ defined by (15), its coefficients provided at page 233 (in reality, equation (15) must be multiplied by 10 to recover the curve of figure 256).

Problem 4.12 of [13] yields a table showing that for a heat flux going from $10^{8}$ to $10^{9} \mathrm{~W} / \mathrm{m}^{2}, T_{t}$ goes from 27 to $317 \mathrm{eV}$. In figure A2, $I\left(T_{t}\right) / T_{t}$ varies by only $9 \%$ about its mean value for $100<T_{t} \leq 300 \mathrm{eV}$, and may be considered as almost constant. However, for $T_{t} \leq 100 \mathrm{eV}$ it is a rapidly increasing function of $T_{t}$.

\section{Appendix B. Nonlinear $A\left(P_{t}\right)$}

The end of the previous Appendix shows that for tungsten, $I\left(T_{t}\right) / T_{t}$ is a rapidly increasing function of $T_{t}$ for $T_{t} \leq 100 \mathrm{eV}$. Therefore, for the values of $P_{t}$ corresponding to this range of temperatures, $A\left(P_{t}\right)$ must be considered as a nonlinear function. The 


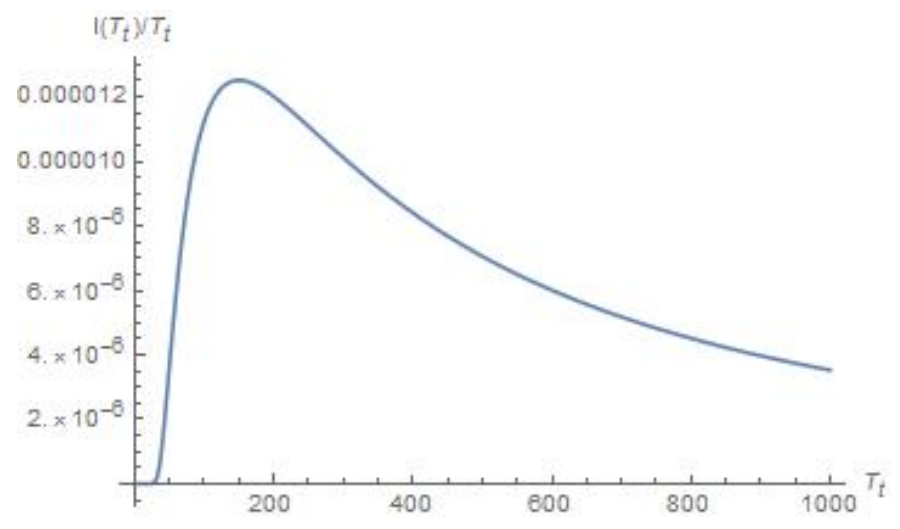

Figure A2. $I\left(T_{t}\right) / T_{t}$ for physical sputtering of tungsten by deuterons.

explicit dependence of $T_{t}$ on $P_{t}$ is not known, but we can get a rough estimate of $A\left(P_{t}\right)$ for $T_{t} \leq 100 \mathrm{eV}$, by assuming that $A\left(P_{t}\right)=\beta P_{t}^{2}$, where $\beta$ is a constant.

Then, (6) becomes

$$
R_{+}=\beta(P-R)^{2} .
$$

This yields $R_{e q}=P+[1-\sqrt{1+4 \beta P}] / 2 \beta$. The linearization of (B.1) for $R$ close to $R_{e q}$ provides $R_{+}-R_{e q}=-\alpha^{\prime}\left(R-R_{e q}\right)$, where $\alpha^{\prime}=2 \beta\left(P-R_{e q}\right)$. The instability now corresponds to $\alpha^{\prime}>1$, that is $\beta P>3 / 4$, or

$$
\alpha>3 / 4 \frac{P-R_{e q}}{P}
$$

by combining (7) and (B.1). This threshold in $\alpha$ is lower than 1, which makes the instability easier than for a linear $A\left(P_{t}\right)$. This kind of reasoning can be generalized for any growing function $A\left(P_{t}\right)$. We finally note that the existence of two basins of PWSO does not depend on the precise dependence of $A$ upon $P_{t}$.

\section{Appendix C. One-dimensional description}

We now introduce a one-dimensional iterative model of PWSO, which makes more explicit the physical mechanisms underlying the 0-dimensional instability. It couples an impurity transport equation and a heat transport equation, which are respectively of the kind used in the Engelhardt and Feneberg model, and in the Zanca et al. model. As in many studies about plasma-wall interaction [13], we use a slab model to describe the radial direction of the plasma.

\section{Appendix C.1. One-dimensional model}

Keeping in mind our initial cylindrical picture, the number of plasma ions/neutrals impacting the target per unit time is

$$
N_{t}(t)=-\frac{2 \pi a L K \partial_{x} T\left(r_{L C F S}, t-\tau_{S O L}\right)+R_{S O L}}{T_{t}},
$$


where

$$
r_{L C F S}=a-\lambda_{S O L}
$$

is the position of the LCFS, $\tau_{S O L}$ is the propagation time of heat from the LCFS to the targets, $K$ is a uniform effective perpendicular conductivity, and $R_{S O L}$ is the power radiated in the SOL, which diminishes both $P_{t}$ and $T_{t}$; it is taken as constant as yet. $R_{S O L}$ and $T_{t}$ depend on the SOL regime $([13,17])$. Then, the number of sputtered atoms per unit time on the two targets is

$$
s(t)=N_{t}(t) I\left(T_{t}(t)\right)
$$

In our slab model, position $x$ is a proxy for the small radius. It spans $[0, a]$ where $a$ is the wall radius. We assume the plasma density $n$ to be uniform.

In our initial cylindrical model, the produced impurities are distributed over an area $2 \pi(a-\lambda) L$, where $\mathrm{L}$ is the perimeter related to the large radius of the reference toroidal system. In the slab model this yields an impurity source term

$$
S(t)=f \frac{s\left(t-\tau_{\text {sput }}\right)}{2 \pi(a-\lambda) L} \delta(x-a+\lambda),
$$

where $\delta$ is the Dirac function and $\tau_{\text {sput }}$ is the flight time of sputtered atoms to the ionization position $a-\lambda$.

We consider a one-dimensional model coupling the impurity transport equation and the heat transport equation. Taking into account equations (C.1, C.3, C.4), the impurity transport equation is

$$
\partial_{t} n_{i}-D \partial_{x}^{2} n_{i}=C_{i}\left[\partial_{x} T\left(r_{L C F S}, t-\tau_{\text {delay }}\right)+T_{l o s s}^{\prime}\right] \delta(x-a+\lambda),
$$

where $n_{i}(x)$ is the impurity density, $D$ is a uniform diffusion coefficient, $T_{\text {loss }}^{\prime}=$ $R_{S O L} /(2 \pi a L K)$ corresponds to a decrease of the thermal flux on the targets,

$$
C_{i}=-\frac{f a K I\left(T_{t}\right)}{(a-\lambda) T_{t}}
$$

and

$$
\tau_{\text {delay }}=\tau_{\text {sput }}+\tau_{S O L}
$$

We assume the time evolution to be slow enough for the inductive contribution of the electric field to be negligible. Using the corresponding simple Ohm's law, the heat transport equation is

$$
n \partial_{t} T-K \partial_{x}^{2} T=C_{T} T^{3 / 2}+p_{a d d}-n n_{i} \operatorname{Rad}(\mathrm{T})
$$

where $T$ is the temperature, $p_{\text {add }}$ is the additional power density, $\operatorname{Rad}(\mathrm{T})$ is the impurity radiation rate coefficient, and

$$
C_{T}=\frac{E_{0}^{2}}{\eta(T) T^{3 / 2}} \simeq 6.510^{2} \frac{E_{0}^{2}}{Z} .
$$

with $E_{0}$ the electric field corresponding to the loop voltage, $Z$ the charge number of the main ion, SI units are used, and $\eta(T)$ the transverse Spitzer resistivity, as given in page 29 of [42] where the Coulomb logarithm was taken as equal to 15, as in [8, 9]. 
Appendix C.2. Iterative equation

We consider a state of the system defined by equations (C.5) and (C.8) close to an equilibrium.

Appendix C.2.1. Large diffusivity limit We first assume $D$ and $K$ to be large enough for the time derivatives to be negligible. Integrating equation (C.5) over the interval $[0, x]$ and assuming $\partial_{x} n_{i}(0)=0$, then integrating again over the interval $[x, a]$ and assuming $n_{i}(a)=0$ yields

$$
D n_{i}(x, t)=-C_{i} l(x)\left[T^{\prime}\left(r_{L C F S}, t-\tau_{\text {delay }}\right)+T_{\text {loss }}^{\prime}\right],
$$

where $T^{\prime}=\partial_{x} T, l(x)=\lambda$ for $x \leq a-\lambda$ and $l(x)=a-x$ for $x>a-\lambda$. Integrating equation (C.8) over $\left[0, r_{L C F S}\right]$, using equation (C.10), and assuming $\partial_{x} T(0)=0$ yields

$$
\begin{aligned}
-K \partial_{x} T\left(r_{L C F S}\right) & =\int_{0}^{r_{L C F S}}\left[C_{T} T(x)^{3 / 2}+p_{a d d}(x)\right] \mathrm{d} x \\
& +\frac{n C_{i}}{D}\left[T^{\prime}\left(r_{L C F S}, t-\tau_{\text {delay }}\right)+T_{\text {loss }}^{\prime}\right] \lambda \lambda_{\text {rad }} \operatorname{Rad}_{\text {max }},
\end{aligned}
$$

where we assumed for simplicity radiation to be localized at $a-\lambda$ with a width $\lambda_{\text {rad }}$ and an amplitude $\operatorname{Rad}_{\max }$. As a result,

$$
P_{L C F S}(t)=P+\alpha R_{S O L}-\alpha P_{L C F S}\left(t-\tau_{\text {delay }}\right),
$$

where

$$
\alpha=n \frac{C_{i} \lambda}{D K} \lambda_{r a d} \operatorname{Rad}_{m a x}=\frac{f a \lambda \lambda_{\text {rad }}}{(a-\lambda) D} \frac{n I\left(T_{t}\right)}{T_{t}} \operatorname{Rad}_{\text {max }},
$$

$P_{L C F S}=-a^{\prime} L K T^{\prime}\left(r_{L C F S}\right)$ is the power flowing through the LCFS, and

$$
P=a^{\prime} L \int_{0}^{r_{L C F S}}\left[C_{T} T(x)^{3 / 2}+p_{a d d}(x)\right] \mathrm{d} x,
$$

is the total input power, with $a^{\prime}$ a distance of order $a$ corresponding to an extension of the plasma perpendicular to the $x$ axis, introduced to produce volume integrals. In the integral, $T(x)$ is taken equal to its equilibrium value.

Let

$$
R=P-P_{L C F S}(t)+R_{S O L},
$$

be the total radiated power. Then, (C.12) is equivalent to

$$
R+=R_{S O L}+\alpha(P-R),
$$

where $R+$ is $R$ at time $t+\tau_{\text {delay }}$. We recover the equation obtained in section 4.2 for radiating contributions independent from the target impurities. The simplest case is recovered for $R_{S O L}=0$. If $R_{S O L}$ is not constant, but may be considered as a function of $P-R$, one enters the generalized models already discussed in section 4.1 where $A\left(P_{t}\right)$ departs from a linear dependence on $P_{t}$. One can also add it a term like $R_{0}$ as done for other contributions in this section.

Making the same assumption for the radiation in equation (5) as in equation (C.12), the ratio of the one-dimensional $\alpha$ (C.13) to the zero-dimensional one (7) is $a^{2} D_{\perp} / D(a-\lambda)^{2}$. It is close to 1 by taking identical diffusion coefficients in the two 
models, and by accounting for $\lambda \ll a$. Therefore, the same instability threshold is predicted by both descriptions. Vanishing sputtering at low $T_{t}$ is more visible in the second one, since $T_{\text {loss }}^{\prime}$ increases when approaching detachment. In the second one, the instability bears upon $T^{\prime}\left(r_{L C F S}\right)$, i.e. the thermal flux at the LCFS, but total radiation is slaved to it and becomes unstable concomitantly.

Appendix C.2.2. Moderate diffusivities A moderate value of the diffusion coefficient $D$ sets a delay in the establishment of the stationary profile of equation (C.10). Since it involves the boundary condition $\partial_{x} n_{i}(0)=0$ at $x=0$, a delay time $(a-\lambda)^{2} / D$ is involved to get the new stationary profile. Similarly, if heat diffusivity $\chi$ is moderate, a delay time $(a-\lambda)^{2} / \chi$ is involved to get the new stationary profile. These two delays require substituting equation $\mathrm{C} .7$ with

$$
\tau_{\text {delay }}=\tau_{\text {sput }}+\tau_{S O L}+\frac{(a-\lambda)^{2}}{\chi}+\frac{(a-\lambda)^{2}}{D} .
$$

[1] Greenwald M 2002 "Density limits in toroidal plasmas" Plasma Phys. Control. Fusion 44 R27

[2] Huber A et al 2013 "Impact of the ITER-like wall on divertor detachment and on the density limit in the JET tokamak", J. Nucl. Mater. 438 S139

[3] Duesing G and the JET Team 1986, "First results of neutral beam heating on JET", Plasma Phys. Control. Fusion 281429

[4] Kamada Y, Hosogane N, Yoshino R, Hirayama T and Tsunematsu T 1991 "Study of the density limit with pellet fuelling in JT-60" Nucl. Fusion 311827

[5] Stabler A et al 1992 "Density limit investigations on ASDEX Nucl. Fusion 321557

[6] Mertens V et al 1997 "High density operation close to Greenwald limit and H mode limit in ASDEX upgrade" Nucl. Fusion 371607

[7] Rapp J et al 1999 "Density limits in TEXTOR-94 auxiliary heated discharges" Nucl. Fusion 39 765

[8] Zanca P, Sattin F, Escande D F and JET Contributors 2019 "A power-balance model of the density limit in fusion plasmas: application to the L-mode tokamak" Nucl. Fusion 59126011

[9] Zanca P, Sattin F, Escande D F, Pucella G and Tudisco O 2017 "A unified model of density limit in fusion plasmas" Nucl. Fusion $\mathbf{5 7} 056010$

[10] Zanca P, Sattin F, Escande D F and JET Contributors 2021 "A power-balance model of L-mode density limit in fusion plasmas" to be published

[11] Fuchert G et al "Density related operational limit in the limiter phase of Wendelstein 7-X" 2017 European Conference on Circuit Theory and Design (ECCTD). IEEE, 2017.

[12] Fuchert G et al 2018 "Global energy confinement in the initial limiter configuration of Wendelstein 7-X", Nucl. Fusion $\mathbf{5 8} 106029$

[13] Stangeby P C 2000 The plasma boundary of magnetic fusion devices Vol. 224 (Philadelphia, Pennsylvania: Institute of Physics Pub.)

[14] Yamamura Y and Tawara H (1996) "Energy dependence of ion-induced sputtering yields from monatomic solids at normal incidence" Atomic data and nuclear data tables 62149

[15] Bartiromo R 1998 "Plasma rotation and finite Larmor radius losses in a reversed field pinch" Phys. Plasmas, 53342

[16] McLean A G et al 2015 "Electron pressure balance in the SOL through the transition to detachment" J. Nucl. Mater. 463533

[17] Krasheninnikov S I and Kukushkin A S 2017 "Physics of ultimate detachment of a tokamak divertor plasma" J. Plasma Phys. 83155830501

[18] Groth $\mathrm{M}$ et al 2013 "Impact of carbon and tungsten as divertor materials on the scrape-off layer conditions in JET" Nucl. Fusion, 53093016 
[19] Garbet X et al 2018 "Synergetic effects of collisions, turbulence and sawtooth crashes on impurity transport" IAEA-CN-234-0093

[20] Lowry C G, Campbell D J, Gottardi N, Lawson K and Vlases G 1990 "Density limits in JET with beryllium" Europhysics Conference Abstracts $\mathbf{1 4} 339$ http://libero.ipp.mpg.de/libero/PDF/EPS_17_Vol1_1990.pdf

[21] Campbell D S 1990 "Confinement and stability in JET: recent results" Plasma Phys. Controlled Fusion 32949

[22] Giannone L et al 2000 "Physics of the density limit in the W7-AS stellarator" PlasmaPphys. Controlled Fusion $\mathbf{4 2} 603$

[23] Greenwald M et al 2014 "20 years of research on the Alcator C-Mod tokamak" Phys. Plasmas 21 110501

[24] Stacey W M 1997 "Density limits in tokamaks" Phys. Plasmas 41069

[25] White R B, Gates D A and Brennan D P 2015 "Thermal island destabilization and the Greenwald limit" Phys. Plasmas 22022514

[26] Spizzo G, private communication.

[27] Hajjar R, Diamond P H and Malkov M 2018 "Dynamics of zonal shear collapse with hydrodynamic electrons" Phys. Plasmas 25062306

[28] Diamond P H, Singh R, Malkov M, Hajjar R, Tynan G, Long T and Ke R "Transport Physics of the Density Limit" TH/P5-5, 28th IAEA Fusion Energy Conference, May 2021

[29] Hong R et al "Edge shear flows and particle transport near the density limit of the HL-2A tokamak" Nuc. Fusion 58016041

[30] Klinger T et al 2019 "Overview of first Wendelstein 7-X high performance operation" Nucl. Fusion 59112004

[31] Wolf R C et al. 2019 "Performance of Wendelstein 7-X stellarator plasmas during the first divertor operation phase" Phys. Plasmas 26082504

[32] Laqua H. private communication

[33] Yoo M-G et al 2018 "Evidence of a turbulent ExB mixing avalanche mechanism of gas breakdown in strongly magnetized systems" Nature Comm. 93523

[34] Mueller D 2013 "The physics of tokamak start-up" Phys. Plasmas 20058101

[35] Coda S et al 2019 "Physics research on the TCV tokamak facility: from conventional to alternative scenarios and beyond" Nucl. Fusion 59112023

[36] Stober J et al 2011 "ECRH-assisted plasma start-up with toroidally inclined launch: multi-machine comparison and perspectives for ITER" Nucl. Fusion 51083031

[37] Podestà M, Fasoli A, Labit B, McGrath M, Müller S H and Poli F M 2005 "Plasma production by low-field side injection of electron cyclotron waves in a simple magnetized torus" Plasma Phys. Control. Fusion 471989

[38] Podestà M, Fasoli A, Labit B, McGrath M, Müller S H and Poli F M 2006 "Experimental characterization and modelling of the particle source in an Electron-Cyclotron wave driven toroidal plasma" Plasma Phys. Control. Fusion 481053

[39] Jackson G L, Politzer P A, Humphreys D A, Casper T A, Hyatt A W, Leuer J A, Lohr J, Luce T C, Van Zeeland M A and Yu J H 2010 "Understanding and predicting the dynamics of tokamak discharges during startup and rampdown" Phys. Plasmas 17056116

[40] Escande D F, "Toward tokamaks with higher densities?", https://hal.archives-ouvertes.fr/hal02929482

[41] Wenninger R et al 2017 "The DEMO wall load challenge" Nucl. Fusion 57046002

[42] Richardson A S 20192019 NRL Plasma Formulary US Naval Research Laboratory 